\title{
Le sesse del lago di Como
}

\author{
Parte II (*)
}

\section{OSCILLAZIONI INTERESSANTI L'INTERO LAGO \\ P. Caloi - M. C. Spadea}

ricevuto il 30 settembre 1959

\section{I - STIMA APPROSSTMATIVA DEI PERIODI DI ONDE STAZIONARIE IN UN SISTEMA DI TRE CANALI CHIUSI.}

Il lago di Como ha una forma caratteristica, che si può approssimare a quella di un canale (parte settentrionale), che si biforca all'altezza di Bellagio in due canali, aventi gli estremi l'uno (occidentale) a Como e l'altro a Lecco (di dove esce l'Adda).

Indichiamo con $b_{1}, b_{2}, b_{3}$ e $h_{1}, h_{2} h_{3}$ la larghezza media e la profondità media dei tre rispettivi canall presi in considerazione. L'approssimazione che così si ottiene è tanto più attendiblle quanto più le larghezze $\begin{array}{llll}b_{1} & b_{2} & b_{3} & \text { sono piccole rispetto alla lunghezza dei canali; condizione questa }\end{array}$ pienamente soddisfatta nel lago di Como.

In questa ipotesi, l'innalzamento della superficie, sotto l'azione di un'onda lunga passante per ll punto di confluenza dei tre rami, può considerarsi identica in tre sezioni (appartenente ognuna ad uno dei tre rami) il più vicino al punto di confluenza. Per la trattazione matematica, le sezioni considerate possono ritenersi attraversare l'origine delle coordinate di superficie di ogni canale; in tal modo, la sezione 1 può passare attraverso $x_{1}=0$ e la lunghezza $l_{\text {r }}$ dell'intero primo canale viene contata da questa sezione all'estremità del canale stesso.

Il moto di onde lunghe in un canale a sezione uniforme è rappresentato dalle due equazioni differenziali:

$$
\frac{\partial^{2} \xi}{\partial t^{2}}=-g \frac{\partial y}{\partial x} ; \quad \eta=-h \frac{\partial \xi}{\partial x},
$$

(*) Le parti I, II di questo lavoro sono state condotte con contributi del Consiglio Nazionale delle Ricerche. 
dove $\xi$ rappresenta lo spostamento orizzontale delle particelle d'acqua, $\eta$ lo spostamento verticale della superficie nello stesso punto e $h$ la profondità media della sezione trasversale del canale considerato.

Nel caso di movimento armonico semplice di periodo $T=\frac{2 \Pi}{\sigma}$ possiamo porre,

$$
\begin{aligned}
& \xi=\cos \sigma\left(A \cos \frac{\sigma x}{c}+B \sin \frac{\sigma x}{c}\right), \operatorname{con} c=\sqrt{g h} \\
& \eta=\frac{h \sigma}{c} \cos \sigma t\left(A \sin \frac{\sigma x}{c}-B \cos \frac{\sigma x}{c}\right)
\end{aligned}
$$

espressioni che soddisfano le equazioni del moto. Evidentemente $c$ rappresenta la velocità di propagazione di un'onda libera lungo il canale di profondità $h$. Le costanti $A, B, h, c$ e la coordinata $x$ si intendono affette degli indici $1,2,3$, a seconda che si riferiscano al primo canale $o$ alle due ramificazioni, nell'ordine.

Per il punto di confluenza, dove $x_{1}=x_{z}=x_{3}=0$, si ottiene

$$
B_{1} \frac{h_{1}}{c_{1}}=B_{2} \frac{h_{2}}{c_{2}}=B_{3} \frac{h_{3}}{c_{3}}
$$

La condizione di incompressibilità richiede che la quantità d'acqua fluente, in un certo tempo, attraverso la sezione 1 verso la confluenza, uguagli la somma delle quantità attraversanti le sezioni 2,3 , oltre la confluenza. Ciò porta come conseguenza che per $x_{1}=x_{2}=x_{3}=0$, si ha:

$$
\sum_{i}^{3} A_{i} b_{i} h_{i}=0
$$

All'estremità dei canali chiusi, avremo poi

$$
\xi_{i}=0 \quad \text { per } x_{i}=l_{i} . \quad(i=1,2,3)
$$

Dalla [1] consegue allora

$$
\frac{A_{i}^{\prime}}{B_{i}}=-\operatorname{tg} \frac{\sigma l}{c_{i}} . \quad(i=1,2,3)
$$

Dalla [1], ricordando la [4], otteniamo

$$
\xi_{i}=B_{i} \frac{\cos \sigma t}{\frac{\cos \sigma l_{i}}{c_{i}}} \sin \frac{\sigma\left(x_{i}-l_{i}\right)}{c_{i}},(i=1,2,3)
$$


Tabella I

\begin{tabular}{|c|c|c|c|c|c|c|c|}
\hline \multicolumn{4}{|c|}{ LECCO-BELLAGIO $\quad l_{2}=19.000 \mathrm{~m}$} & \multicolumn{4}{|c|}{ Como-Bellagio $\quad l_{1}=23.375 \mathrm{~m}$} \\
\hline Sez. & $\begin{array}{c}S(x) \\
10^{2} \mathrm{~m}^{2}\end{array}$ & $\begin{array}{c}b(x) \\
10^{2} \mathrm{~m}\end{array}$ & $\begin{array}{c}h \\
\mathrm{~m}\end{array}$ & Sez. & $\begin{array}{c}S(x) \\
10^{2} \mathrm{~m}^{2}\end{array}$ & $\begin{array}{c}b(x) \\
10^{2} \mathrm{~m}\end{array}$ & $\begin{array}{l}h \\
\mathrm{~m}\end{array}$ \\
\hline $\begin{array}{c}0^{\mathrm{a}} \\
1^{\mathrm{a}} \\
2^{\mathrm{a}} \\
3^{\mathrm{a}} \\
4^{\mathrm{a}} \\
5^{\mathrm{a}} \\
6^{\mathrm{a}} \\
7^{\mathrm{a}} \\
8^{\mathrm{a}} \\
9^{\mathrm{a}} \\
10^{\mathrm{a}} \\
11^{\mathrm{a}} \\
12^{\mathrm{a}} \\
13^{\mathrm{a}} \\
14^{\mathrm{a}} \\
15^{\mathrm{a}} \\
16^{\mathrm{a}} \\
17^{\mathrm{a}} \\
18^{\mathrm{a}}\end{array}$ & $\begin{array}{r}0 \\
500 \\
985 \\
1355 \\
1325 \\
1805 \\
1440 \\
1275 \\
1870 \\
1420 \\
1065 \\
1945 \\
2585 \\
2790 \\
2810 \\
2925 \\
4170 \\
5710 \\
4100\end{array}$ & $\begin{array}{c}0 \\
8.00 \\
13.75 \\
12.75 \\
11.75 \\
14.25 \\
13.50 \\
12.00 \\
16.50 \\
16.50 \\
14.75 \\
18.25 \\
22.25 \\
19.75 \\
20.00 \\
23.75 \\
26.00 \\
24.50 \\
19.50 \\
\mathrm{~b}=\mathrm{m} 1710.00\end{array}$ & $\begin{array}{r}0 \\
29.25 \\
57.62 \\
79.27 \\
77.51 \\
105.59 \\
84.24 \\
74.59 \\
109.39 \\
83.07 \\
62.30 \\
113.78 \\
151.22 \\
163.21 \\
169.38 \\
171.11 \\
243.94 \\
298.93 \\
239.85 \\
\bar{h}=\mathrm{m} 121.80\end{array}$ & $\begin{array}{r}0 \\
1 \\
2 \\
3 \\
4 \\
5 \\
6 \\
7 \\
8 \\
9 \\
10 \\
11 \\
12 \\
13 \\
14 \\
15 \\
16 \\
17 \\
18 \\
19 \\
20 \\
21 \\
22 \\
23 \\
24 \\
25 \\
26 \\
27\end{array}$ & $\begin{array}{r}0 \\
382.50 \\
972.75 \\
1383.25 \\
1560.75 \\
1875.00 \\
1230.50 \\
1549.75 \\
3770.75 \\
3248.75 \\
2647.25 \\
2168.75 \\
2187.00 \\
4362.50 \\
4892.50 \\
4770.50 \\
4989.50 \\
5663.50 \\
5992.75 \\
5510.25 \\
4900.00 \\
6300.00 \\
4850.00 \\
4869.75 \\
5353.50 \\
2511.50 \\
2383.75 \\
1267.75\end{array}$ & \begin{tabular}{|c|}
0 \\
7.00 \\
11.25 \\
16.00 \\
13.25 \\
16.00 \\
8.25 \\
10.75 \\
18.00 \\
17.50 \\
13.25 \\
9.75 \\
8.00 \\
15.00 \\
17.25 \\
14.75 \\
17.00 \\
20.50 \\
20.25 \\
20.75 \\
23.50 \\
23.50 \\
19.00 \\
24.50 \\
25.75 \\
16.75 \\
16.00 \\
18.09 \\
$b=\mathrm{m} 1623.00$
\end{tabular} & $\begin{array}{r}0 \\
23.56 \\
59.92 \\
85.21 \\
96.14 \\
115.50 \\
75.80 \\
95.46 \\
232.28 \\
200.12 \\
163.07 \\
133.59 \\
134.72 \\
268.73 \\
301.38 \\
293.86 \\
307.35 \\
348.87 \\
369.15 \\
339.43 \\
301.84 \\
388.10 \\
298.76 \\
299.98 \\
329.78 \\
154.71 \\
146.84 \\
78.09 \\
\\
h=\mathrm{m} 201.51\end{array}$ \\
\hline
\end{tabular}

\begin{tabular}{|c|c|c|c|}
\hline \multicolumn{2}{|c|}{$R_{A} M O$} & \multicolumn{2}{|c|}{$l_{3}=28.000 \mathrm{~m}$} \\
\hline \multirow{2}{*}{ Sez. } & $S(x)$ & $b(x)$ & $h$ \\
\hline & $10^{2} \mathrm{~m}^{2}$ & $10^{2} \mathrm{~m}$ & m \\
\hline 28 & 4637 & 40.50 & 155.76 \\
\hline 29 & 6107 & 40.00 & 209.39 \\
\hline 30 & 7818 & 37.00 & 274.70 \\
\hline 31 & 8170 & 33.50 & 289.22 \\
\hline 32 & 9505 & 37.50 & 336.48 \\
\hline 33 & 9330 & 34.25 & 330.28 \\
\hline 34 & 9010 & 37.00 & 318.95 \\
\hline 35 & 7490 & 34.50 & 265.15 \\
\hline 36 & 7530 & 33.50 & 266.56 \\
\hline 37 & 2605 & 20.25 & 92.22 \\
\hline 38 & 2800 & 18.00 & 99.12 \\
\hline 39 & 4340 & 26.50 & 153.64 \\
\hline 40 & 5070 & 28.50 & 179.48 \\
\hline 41 & 5635 & 31.75 & 199.48 \\
\hline 42 & 4730 & 27.50 & 167.44 \\
\hline 43 & 3990 & 22.00 & 191.25 \\
\hline 44 & 3325 & 33.25 & 117.70 \\
\hline 45 & 5325 & 42.50 & 188.50 \\
\hline 46 & 2595 & 20.75 & 91.86 \\
\hline 47 & 2680 & 26.25 & 94.87 \\
\hline 48 & 2965 & 30.75 & 104.96 \\
\hline 49 & 1525 & 25.55 & 53.98 \\
\hline 50 & 235 & 11.00 & 8.32 \\
\hline \multirow[t]{2}{*}{51} & 0 & 0 & 0 \\
\hline & & $b=\mathrm{m} 2822.00$ & $\bar{h}=\mathrm{m} 174.55$ \\
\hline
\end{tabular}


Tabella I I

\begin{tabular}{|c|c|c|c|c|c|c|c|c|c|c|}
\hline \multicolumn{6}{|c|}{ LE C C O - B E L L A G I O } & \multicolumn{3}{|c|}{ C O M $0 \cdot$ B E L L A G I 0} & \multicolumn{2}{|c|}{$l_{1}=23.375 \mathrm{~m}$} \\
\hline Sez. & $\begin{array}{l}a \Delta x \\
10-4\end{array}$ & $\begin{array}{c}q \\
10^{9} \\
\end{array}$ & $\begin{array}{c}2 \xi_{o} \\
10^{3} \mathrm{~cm}\end{array}$ & $\begin{array}{c}2 \triangle \eta_{0} \\
\mathrm{~cm}\end{array}$ & $\begin{array}{l}2 \eta_{0} \\
\mathrm{~cm}\end{array}$ & Sez. & $\begin{array}{c}q \\
10^{9} \\
\end{array}$ & $\begin{array}{c}2 \xi_{0} \\
10^{3} \mathrm{~cm}\end{array}$ & $\begin{array}{c}2 \Delta \eta_{0} \\
\mathrm{~cm}\end{array}$ & $\begin{array}{l}2 \eta_{0} \\
\mathrm{~cm}\end{array}$ \\
\hline 0a & 7.907 & 0 & 0 & 0 & 100 & 0 & 0 & 0 & 0 & 100 \\
\hline la & ) & 825 & $-1,65$ & $-1,30$ & 98,70 & 1 & 1111,25 & $-2,91$ & $-2,30$ & 97,70 \\
\hline $2^{a}$ & " & 2034,07 & $-2,06$ & $-1,63$ & 97,07 & 2 & 1931,93 & $-1,99$ & $-1,57$ & 96,13 \\
\hline $3 \mathbf{a}$ & $n$ & 3344,51 & $-2,47$ & $-1,95$ & 95,12 & 3 & 3104,72 & $-2,24$ & $-1,77$ & 94,36 \\
\hline $4^{\mathrm{a}}$ & " & 4533,51 & $-3,42$ & $-2,70$ & 92,42 & 4 & 4116,73 & $-2,64$ & $-2,09$ & 92,27 \\
\hline $5^{a}$ & $n$. & 5804,28 & $-3,22$ & $-2,55$ & 89,87 & 5 & 5279,33 & $-2,82$ & $-2,23$ & 90.04 \\
\hline $6^{a}$ & n & 7287,13 & $-5,06$ & $-4,00$ & 85,87 & 6 & 6359,81 & $-5,17$ & $-4,09$ & 85,95 \\
\hline $7 a$ & נ & 8424,91 & $-6,61$ & $-5,23$ & 80,64 & 7 & 7172,04 & $-4,63$ & $-3,66$ & 82,29 \\
\hline $8^{a}$ & " & 9715,15 & $-5,20$ & $-4,11$ & 76,53 & 8 & 8519,54 & $-2,26$ & $-1,79$ & 80,50 \\
\hline $9^{a}$ & ” & 11188,35 & $-7,88$ & $-6,23$ & 70,30 & 9 & 10103,38 & $-3,11$ & $-2,46$ & 78,04 \\
\hline $10^{a}$ & ” & 12401,02 & $-11,64$ & $-9,20$ & 61,10 & 10 & 11422,26 & $-4,31$ & $-3,41$ & 74,63 \\
\hline $11 \mathrm{a}$ & ” & 13592,47 & $-6,99$ & $-5,53$ & 55,57 & 11 & 12340,21 & $-5,69$ & -450 & 70.13 \\
\hline $12^{a}$ & & 14828,90 & $-5,74$ & $-44,5$ & 51,03 & 12 & 12859,17 & $-5,88$ & $-4,65$ & 65,48 \\
\hline $13^{a}$ & » & 15977,07 & $-5,73$ & $-4,58$ & 46,50 & 13 & 13612,19 & $-3,12$ & $-2,47$ & 6301 \\
\hline $14^{\mathrm{a}}$ & ” & 16965,19 & $-6,04$ & $-4,73$ & 41,72 & 14 & 14710,14 & $-3,01$ & $\begin{array}{r}2,38 \\
\end{array}$ & 60,63 \\
\hline $15^{a}$ & \# & 17914,32 & $-6,12$ & $-4,84$ & 36,88 & 15 & 15655,97 & $-3,28$ & $-2,59$ & 58,04 \\
\hline $16^{a}$ & " & 18928,52 & $-4,54$ & $-3,59$ & 33,29 & 16 & 16518,59 & $-\mathbf{3}, \mathbf{3 1}$ & $-2,62$ & 55,42 \\
\hline $17^{a}$ & $"$ & 19744,12 & $-3,46$ & $-2,74$ & 30,55 & 17 & 17633,92 & $-3,11$ & $-2,46$ & 52,96 \\
\hline $18^{a}$ & ” & 20500,23 & $-5,00$ & $-3,95$ & 26,60 & 18 & 18746,08 & $-3,13$ & $-2,47$ & 50,49 \\
\hline $19^{a}$ & " & 21072,13 & $-4,79$ & $-3,79$ & 22,81 & 19 & 19806,37 & $\begin{array}{r}3,59 \\
-3\end{array}$ & $-2,84$ & 47,65 \\
\hline & & (a) & & . & & 20 & 20832,04 & $-4,25$ & $-3,36$ & 44,29 \\
\hline & & & & & & 21 & 21971,40 & $-3,49$ & $-2,76$ & 41,53 \\
\hline & & & & & & 22 & 22926,59 & $-4,73$ & $-3,74$ & 37,79 \\
\hline & & & & & & 23 & 23662,55 & $-4,86$ & $-3,84$ & 33,95 \\
\hline & & & & & & 24 & 24490,08 & $-4,57$ & $-3,61$ & 30,34 \\
\hline & & & & & & 25 & 25142,39 & $-10,01$ & $-7,91$ & 22,43 \\
\hline & & & & & & 26 & 25533,23 & $-10,71$ & $-8,47$ & 13,96 \\
\hline & & & & & & 27 & 25799,52 & $-20,35$ & $-16,09$ & $-2,13$ \\
\hline
\end{tabular}

\begin{tabular}{|c|c|c|c|c|}
\hline \multicolumn{5}{|c|}{ RAMO VNICO } \\
\hline Sez. & $\begin{array}{c}q \\
10^{9}\end{array}$ & $\begin{array}{c}2 \xi_{0} \\
10^{3} \mathrm{~cm}\end{array}$ & $\begin{array}{c}2 \Delta \eta_{0} \\
\mathrm{~cm}\end{array}$ & $\begin{array}{l}2 \eta_{0} \\
\mathrm{~cm}\end{array}$ \\
\hline & 23772,00 & 0 & 0 & $-\quad 2,13$ \\
\hline 28 & 23687,55 & $-5,11$ & $-4,04$ & 6,17 \\
\hline 29 & 23466,23 & $-3,84$ & $-3,04$ & $-\quad 9,21$ \\
\hline 30 & 23067,44 & $-2,95$ & $-2,33$ & $-11,54$ \\
\hline 31 & 22609,65 & $-2,77$ & $-2,19$ & $-13,73$ \\
\hline 32 & 22046,72 & $-2,32$ & $-1,83$ & $-15,56$ \\
\hline 33 & 21397,09 & $-2,29$ & $-1,81$ & $-17,37$ \\
\hline 34 & 20789,14 & $-2,31$ & $-1,83$ & $-19,20$ \\
\hline 35 & 20073,94 & $-2,68$ & $-2,12$ & $-21,32$ \\
\hline 36 & 19274,44 & $-2,56$ & $-2,02$ & $-23,34$ \\
\hline 37 & 18550,90 & $-7,12$ & $-5,63$ & $-28,97$ \\
\hline 38 & 18029,44 & $-6,44$ & $-5,09$ & $-34,06$ \\
\hline 39 & 17177,94 & $-3,96$ & $-3,13$ & $-37,19$ \\
\hline 40 & 16193,11 & $-3,19$ & $-2,52$ & $-39,71$ \\
\hline 41 & 14882,68 & $-2,64$ & $-2,09$ & $-41,80$ \\
\hline 42 & 13388,33 & $-2,83$ & $-2,24$ & $-44,04$ \\
\hline 43 & 11890,97 & $-2,98$ & $-2,36$ & $-46,40$ \\
\hline 44 & 10487,37 & $-3,15$ & $-2,49$ & $-48,99$ \\
\hline 45 & 8409,55 & $-1,58$ & $-1,25$ & $-50,14$ \\
\hline 46 & 6529,30 & $-2,52$ & $-1,99$ & $-52,13$ \\
\hline 47 & 5239,08 & $-1,95$ & $-1,54$ & $-53,67$ \\
\hline 48 & 3374,05 & $-1,14$ & $-0,90$ & $-54,57$ \\
\hline 49 & 1586,88 & $-1,04$ & $-0,82$ & $-55,39$ \\
\hline 50 & 396,00 & $-1,69$ & $-1,34$ & $-56,73$ \\
\hline 51 & 112,35 & & & \\
\hline
\end{tabular}


mentre dalla [3] consegue, nel caso dei tre canali chiusi,

$$
\sum_{1}^{3} B_{i} b_{i} h_{i} \operatorname{tang} \frac{\sigma l_{i}}{c_{i}}=0
$$

oppure (v. [2])

$$
\sum_{i}^{3} b_{i} c_{i} \operatorname{tang} \frac{\sigma l_{i}}{c_{i}}=0
$$

È questa l'equazione di Zeilon (').

Ci siamo proposti di applicare, nella determinazione del periodo, approssimativo dell'oscillazione libera uninodale del lago di Como, la formula [5].

Le lunghezze dei tre rami, dei quali questo lago consiste, sono rispettivamente $l_{1}=23.375 \mathrm{~m}$. (Como-Bellagio), $l_{2}=19.000 \mathrm{~m}$. (LeccoBellagio), $l_{3}-28.000 \mathrm{~m}$. (Bellaio-riva); le rispettive larghezze medie sono $b_{1}=2822 \mathrm{~m} ., b_{2}=1710 \mathrm{~m} ., b_{3}-1623 \mathrm{~m}$., come risulta dalla Tabella I. La stessa Tabella fornisce anche le profondità medie, le quali permettono di calcolare i valori delle $c_{i}$ : si è ottenuto $c_{1}=41,36 \mathrm{~m} / \mathrm{sec}$, $c_{2}=34,75 \mathrm{~m} / \mathrm{sec} ., c_{3}=44,44 \mathrm{~m} / \mathrm{sec}$. Con questi dati morfometrici, risolvendo la [5] con il metodo delle approssimazioni successive, si è ottenuto

$$
T=40^{\mathrm{m}}, 6
$$

come periodo dell'uninodale dell'intero lago. Si tratta, naturalmente di un valore approssimativo, che - come vedremo - non differisce molto da quello ottenuto con altro metodo.

II - Il metodo di Defant è stato già esposto in lavori precedenti $\left(^{2}\right)$.

L'applicazione di tale metodo ad un lago ramificato, comporta alcuni accorgimenti da tener presenti per la zona del lago dove avviene la saldatura dei tre rami.

Siano, per esempio, $n_{1}$ il numero delle sezioni del ramo più corto (Lecco), $n_{2}$ il numero delle sezioni dell'altro ramo (Como). Siano inoltre $\alpha$ e $\beta$ i valori di $2 \eta_{0}$, corrispondenti alle sezioni di $n_{1}$ ed $n_{2}$ rispettivamente e siano, inoltre $A$ e $B$ i relativi valori di $q$. Per determinare il valore di $q$ corrispondente alla sezione $n_{1}$, quale sarebbe se $2 \eta_{0}$ fosse uguale a $\beta$, $A$ va moltipllcato per $\beta / \alpha$.

Pertanto il calcolo relativo al ramo nord inizierà con la sezione $n_{1}+n_{2}$, dove $q$ avrà il valore espresso da $B+A \cdot \beta / \alpha$ e $2 \eta_{0}$ avrà ll valore $\beta$. Per le successive sezioni, ll calcolo procederà nel modo abituale. 
Applicato il metodo di Defant con gli avvertimenti sopra detti, dopo alcuni tentativi si è pervenuti ai valori riportati nella Tabella II, corrispondenti ad un periodo

$$
T=37^{\mathrm{m}}, 6
$$

per l'uninodale dell'intero Lago.

III - Il periodo dell'oscillazione uninodale interessante l'intero lago di Como, dovrebbe cadere fra i $38^{\mathrm{m}}$ e i $40^{\mathrm{m}}$.

$\mathrm{E}$ interessante osservare che l'applicazione del metodo di Hidaka, fatta al lago prescindendo dal ramo di Como-Bellagio ha condotto, come si è visto nel lavoro precedente $\left({ }^{3}\right)$, ad un valore di $39^{\mathrm{m}}$ circa. Data la lieve differenza che passa fra il valore dell'uninodale interessante l'intero lago e quello della stessa oscillazione da Gera e Lecco, d:ıl punto di vista idrodinamico possono realizzarsi entrambe le oscillazioni; fatto che non si sarebbe potuto verificare qualora il lago incompleto avesse avuto un periodo dell'uninodale decisamente maggiore di quello dell'intero lago.

Soltanto le osservazioni potranno confermare o meno questi valori ottenuti per via teorica.

\section{RIASSUNTO}

Applicando all'intero lago due metodi diversi abbiamo ottenuto per l'uninodale valori varianti fra $38 \mathrm{~m}$ e $40 \mathrm{~m}$.

Poichè l'oscillazione da Gera a Lecco (vedi parte I) ha un valore di $39 m$ ca., dal punto di vista idrodinamico possono valorizzarsi entrambe le oscillazioni, cioè sia quella interessante l'intero lago che quella limitata al ramo di Lecco e al suo prolungamento.

\section{$A B S T R A C T$}

Applying to the whole Lake two different methods, we have obtained for the uninodal, figures ranging from $38 \mathrm{~m}$ to $40 \mathrm{~m}$.

As the oscillation from Gera to Lecco (see part I) has reached about $39 \mathrm{~m}$, speaking from hydrodynamic point of view, both oscillations can take place, i.e. the one that concerns the whole lake and the other that is limited to the Lecco branch and its extension. 


\section{BIBIJIOGRAFIA}

(1) Zeilon N., On the Seiches of the Gullmar Fjord. Goteborg (1913).

(2) Calor P., Le sesse del Lago di Garda; Parte II, "Annali di Geofisica" (1948).

$\left({ }^{3}\right)$ Caloi P.-Spadea M. C., Le sesse del Lago di Como, Parte I, "Annali di Geofisica ", (1958). 


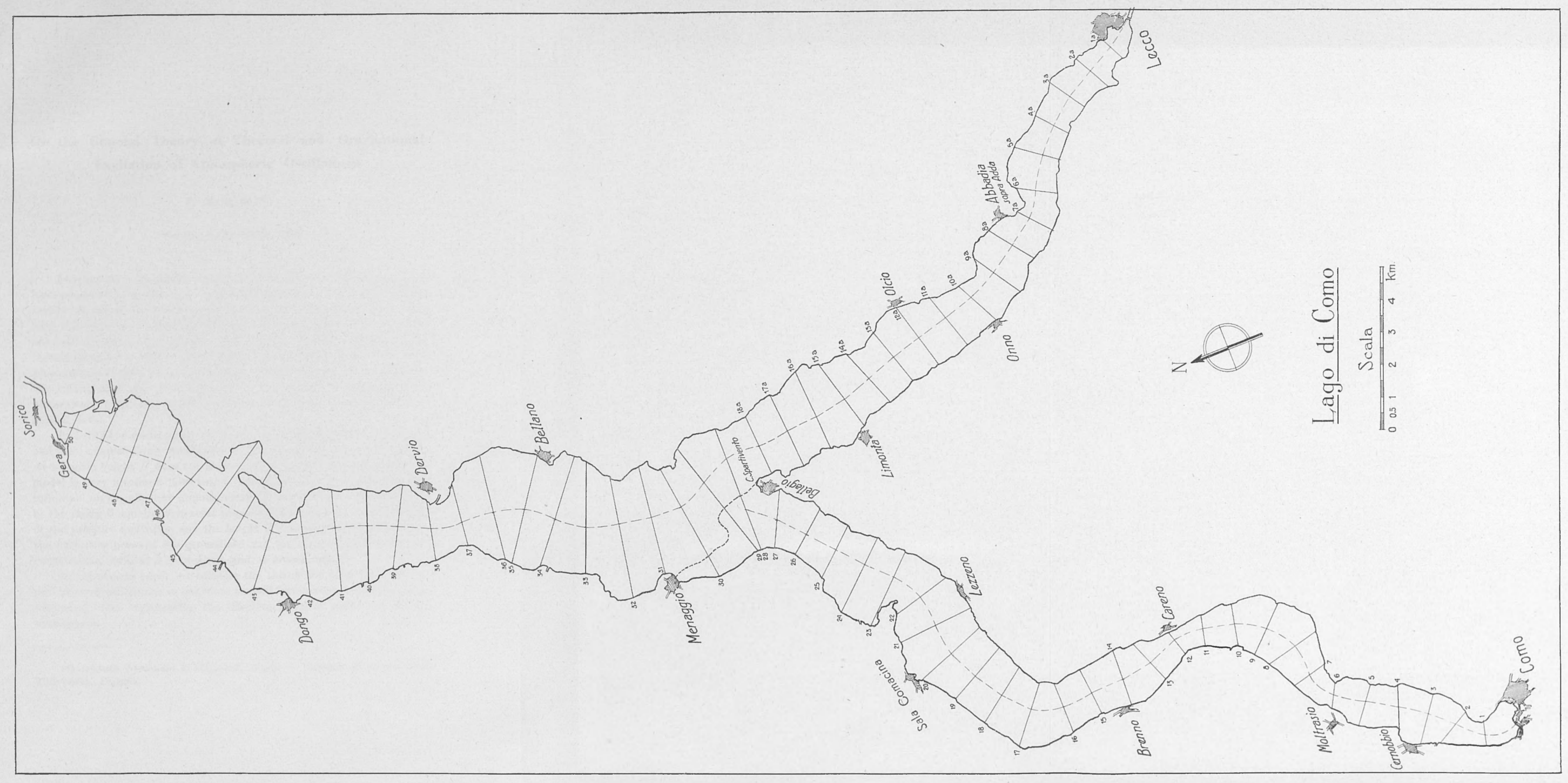

